\title{
Variations
}

Variations

Revue internationale de théorie critique

$23 \mid 2020$

Pour une gauche érotique

\section{La Jazzcogne : territoires et imaginaires de/en lutte}

Dialogue avec Fabien Granjon

\section{Bernard Lubat}

\section{OpenEdition}

Journals

Édition électronique

URL : http://journals.openedition.org/variations/1363

DOI : 10.4000/variations. 1363

ISSN : 1968-3960

Éditeur

Les amis de Variations

Référence électronique

Bernard Lubat, "La Jazzcogne : territoires et imaginaires de/en lutte », Variations [En ligne], 23 | 2020, mis en ligne le 01 septembre 2020, consulté le 07 septembre 2020. URL : http://

journals.openedition.org/variations/1363; DOI : https://doi.org/10.4000/variations.1363

Ce document a été généré automatiquement le 7 septembre 2020

Les ami•e•s de Variations 


\section{La Jazzcogne : territoires et imaginaires de/en lutte}

Dialogue avec Fabien Granjon

\section{Bernard Lubat}

1 Uzeste. Occitanie océanique. Village du Bazadais campé au milieu des forêts de pin des Landes girondines. Sa collégiale, Notre-Dame d'Uzeste, église gothique du XIV ${ }^{\text {ème }}$ siècle abritant le gisant de Clément V (Bertrand de Got), élu au pontificat en 1307, premier des sept papes qui siégèrent à Avignon ; son Café du sport, tenu depuis toujours par des femmes, de mères en filles, haut lieu de la culture ruralo-ouvrière; et bien sûr l'Estaminet-Théâtre Amusicien, à la façade recouverte d'aphorismes d'Antonio Gramsci, d'Édouard Glissant, de William Shakespeare, etc. «N'entrez pas sans désir! », peut-on notamment y lire dans un lettrage dont la taille imposante tranche sensiblement avec la fragilité de la graphie. L'« Estam' », comme on dit à Uzeste, a été fondé en 1937 par les parents de Bernard Lubat: Marie et Alban. Ils quittent le métayage à cette époque et achètent en viager ce lieu dont ils vont faire une espèce de Cabaret Voltaire paysan, où se jouent et se déjouent les contradictions d'une ruralité porteuse de contraintes structurelles, mais aussi d'imaginaires utopiques.

2 Voilà maintenant plus de quarante ans que Bernard Lubat, « mal-poly-instrumentiste », " artiste critique en situation critique " s'est réinstallé dans son village natal pour tenter d'y inventer une ruralité émancipée. En revenant à Uzeste pour y faire la basearrière (à moins que ça ne soit l'avant-poste) de la Compagnie Lubat, il souhaitait redonner quelque fierté à un terroir rural qui dépérissait, notamment culturellement, avec un recul très net de la pratique des traditions et des coutumes, territoire qui lui semblait pourtant toujours riche de potentialités et d'une histoire qu'il s'agissait de se réapproprier et de mettre à l'œuvre autrement que par la mise sous cloche de traditions à préserver du changement. Aussi, son idée fut de faire d'Uzeste un lieu au sens où l'entend Glissant, i.e. un espace de questionnement tant esthétique que politique, ouvert sur le Tout-Monde, afin de maintenir «à vif» (et donc «à vivre) imaginaires d'autonomie et subjectivités rebelles. Et, de fait, par ce travail laborieux au long cours, c'est le territoire uzestois qui s'est trouvé quelque peu désincarcéré, tant 
des aliénations passéistes que des vicissitudes d'un capitalisme toujours plus enclin à exploiter la moindre parcelle de vie.

3 Fabien Granjon - Quand tu es revenu à Uzeste au début des années 1980, après en être parti pendant près de vingt ans, tu as retrouvé un village qui n'était plus l'ombre que de lui-même, que la vie était en train de quitter. Le processus de différenciation sociale qui construisait jusqu'alors la singularité uzestoise - il se dit parfois, encore, que les Uzestois.e.s possèdent deux rangées de dents pour signifier qu'ils ne se laissent pas marcher sur les pieds - s'épuisait sous le double coup de l'exode rural et du développement du monde marchand. La spécificité de la culture populaire «d'ici d'en bas », comme tu le dis souvent, celle des savoir-faire traditionnels et professionnels, des sociabilités villageoises, des valeurs communautaires (notamment l'entraide), de la langue (le parler gascon), des lieux de vie rythmant la quotidienneté (cercle, cafés, Estaminet, entreprises, commerces) qui était à même de dessiner les contours d'une identité locale particulière avait déjà perdu l'essentiel de sa force de distinction et de communalisation. Cette disgrâce s'accompagnait par ailleurs d'une marginalisation symbolique, d'un évident affaissement des imaginaires positifs liés au territoire.

Bernard Lubat - Quand je suis revenu m'installer, Uzeste était un village dont la mémoire s'était arrêtée, dont l'histoire avait pris fin. Il m'a été très difficile d'accepter que cet endroit, à partir duquel j'avais pu faire mon histoire personnelle, parce qu'il y avait une histoire collective et qu'il y avait plein d'histoires auxquelles s'arrimer, fût tout bonnement en train de disparaître. Donc à mon retour, je me suis confronté à l'état dans lequel se trouvait alors mon village natal, son silence, à la manière dont j'avais envie de l'habiter, mais aussi à l'état dans lequel Uzeste se trouvait en moi et m'habitait. À un moment de mon parcours, j'ai pensé que ma place, celle à partir de laquelle je pouvais apporter quelque chose à la dynamique d'émancipation, était nécessairement liée à cette position rurale minoritaire. Ce que je souhaitais créer, un patrimoine contemporain vivant et critique, était en rapport avec les capacités et les incapacités de cette ruralité qui était mienne, tout en m'étant devenue étrangère. Ma légitimité, ma dignité, c'était ma culpabilité et ce que j'étais capable ou pas d'en faire après avoir passé près de vingt ans à Paris dans le milieu du showbiz. Revenir, c'était déjà montrer la valeur du village, mais étonnamment à première vue, ce n'était pas très bien perçu dans la mesure où ça allait à l'encontre du sentiment de honte que beaucoup éprouvaient. Ne plus être d'accord avec cette honte, évidemment, ça engage : à faire le bilan, à rompre, à faire autrement, à résoudre des contradictions qui font mal, à ouvrir sa gueule. Il a fallu que je fasse cet effort de ne pas me laisser taire. Or quand tu arrives avec des idées nouvelles, on essaie d'abord de te faire dégager. À Uzeste, la chose s'est déroulée aussi de cette manière, mais le réflexe traditionnaliste a été rendu un peu plus compliqué, dans la mesure où je suis né là, je comprends et parle le gascon et mes parents ont marqué l'histoire du village en montant L'Estaminet. Donc ce n'est pas simple de me traiter comme un simple étranger. Je suis peut-être un étranger, mais du dedans. Les gens de ma génération ne savent toujours pas comment me considérer. Ils entretiennent, à mon égard, un sentiment mélangé qui tient du refus, mais aussi d'une espèce de respect pour celui d'ici qui, justement, a fait autrement, dont la singularité gênante porte tout de même quelque chose "d'ici d'en bas ", de la misère d'ici, de l'impossibilité d'ici, de cette humanité. Il y a en permanence ce double mouvement de reconnaissance et de peur-répulsion. 
5 F. G. - Pour réussir ta " ré-intégration », tu as dû montrer que ta démarche s'inscrivait dans cette culture populaire de subsistance et de résistance liée au monde rural agricole qui avait fait vivre Uzeste pendant des siècles, dans cette culture de cultivateurs. Aussi, tu as développé une culture radicale puisant dans les racines de la territorialité uzestoise, mais ne s'y résumant pas. La tendance à la capitalisation territoriale à partir d'identités culturelles figées, y compris comme fondement de l'émergence de luttes sociales et d'espaces oppositionnels, n'est assurément pas l'option que tu as choisie. Aux célébrations patrimoniales, à l'engourdissement culturel et aux ressassements communautaro-folkloristes vous avez plutôt répondu par la créolisation, la Relation, le lyannaj (Glissant). Les racines sont importantes, mais considérées comme des ressources " digenésiques ", dont la valeur éventuelle tient à ce qu'elles permettent de s'ouvrir à la rencontre et non de consacrer un plus bas dénominateur commun. Comme l'affirme Édouard Glissant: "L'idée de changer en échangeant est plus importante que l'idée de légitimer en préservant» (2002: 91). Le Même et l'Être ne valent que s'ils sont capables de Divers et il faut comprendre que cette transformation ne se réalise pas toute seule, elle est une lutte politique.

6 B. L. - Le combat est effectivement politique. Je suis revenu à Uzeste pour y cultiver la culture, mais sans travailler à faire de l'identique, car faire de l'identique c'est évidemment une manière de calmer les foules. Les rituels de la tradition doivent être rejoués car à défaut, cela devient vite de la commémoration ankylosante pour con(s-)sacrés. Quand nous reprenons le carnaval ou la fête du printemps, nous nous inspirons de formes archaïques qui entretiennent un fort et très ancien lien avec le lieu. Ces traditions nous rattachent au mouvement de l'histoire, mais quand nous nous les approprions, il s'agit moins d'une célébration mémorielle que d'une critique respectueuse. Nous y ajoutons des éléments qui viennent d'autres traditions, par exemple de la critique sociale: l'interpellation, la manifestation, le slogan, le mégaphone, etc. C'est de cette façon que nous avons contribué à redresser le village et à tenter de le réinscrire dans une ruralité active rendant de nouveau possible un avenir local dialectisant passé et futur, tradition et innovation. Nous avons développé une nouvelle culture pouvant redonner quelque fierté à cet univers rural moribond et mortifère, marqué par la disparition de ses agriculteurs, de ses éleveurs, de sa langue, de ses coutumes, de sa solidarité paysanne, l'exil de sa jeunesse. Depuis notre place d'artistes, nous remettons les pendules à l'œuvre et tentons de maintenir un contrat d'engagement social pour un futur à vivre, à conquérir, à construire, à partager avec abnégation, courage, compétence, patience, et souvent avec souffrance devant certaines indifférences. Nous essayons de montrer qu'une vie n'est bien remplie que si l'on comprend qu'il faut précisément tout une vie durant s'y apprendre, s'y cultiver, s'y interroger, s'y instruire, s'y critiquer, s'y inventer sans cesse de la naissance à la mort. C'est là notre responsabilité, notre mandat, que de montrer que «d'ici d'en Sud Gironde » peuvent se façonner de vrais artistes citoyens concernés, impliqués, engagés qui respirent la liberté, la sensibilité, l'imagination, la responsabilité, ouverts sur le monde, sur l'autre, sur le différent(d) autant que sur le référent, dans le respect de l'héritage qui est ici le notre.

7 F. G. - Autour d'Uzeste, émergent depuis des années nombre d'initiatives alternatives et d'espaces de solidarité (maraîchage en permaculture, habitat coopératif, événements culturels, réouverture de cercles, etc.) dont les instigateurs déclarent quasi systématiquement entretenir une dette assumée envers toi, la Compagnie Lubat, les 
hestejadas de las arts et votre projet de Visage village des arts à l'oeuvre. Ce dû n'est aucunement d'ordre matériel ou financier et ne s'inscrit pas non plus nécessairement dans une filiation disciplinaire - les arts, le jazz -, mais relève plutôt d'un legs "encapacitatoire", d'un désir de faire qui s'actualise donc parfois en un pouvoir effectif (d')agir et de création. Vous avez, me semble-t-il, réussi, au moins pour partie, à re-innerver et à réinventer le territoire en ses entours en développant des «systèmes d'échanges » singuliers, plus ou moins formalisés, avec d'autres espaces que l'on pourrait qualifier d'«uzestiens », c'est-à-dire affectés par les imaginaires uzestois et leur pouvoir instituant. Ces lieux apparaissent comme des espèces de «décrets d'application " de ce devoir d'invention d'une ruralité critique que tu évoques souvent. Ils sont des foyers de relations sociales alternatives, des lieux d'institution d'une praxis instituante qui porte ce que Pascal Nicolas-Le Strat appelle un travail du commun (2016), un travail de "ré-institution générale de l'existence collective » (Laval et al., 2019: 8), lequel se trouve être nécessairement situé/localisé dans ses réalisations pratiques liées à des ressources territoriales, mais fortement déterritorialisable en son principe.

B. L. - Il s'agit d'essayer de créer ce qui est profitable à toutes et tous et sera heureux à nos descendants. Peut-on changer la société qui nous entoure en choisissant de transformer la norme, dans le domaine artistique comme dans le domaine politique ou économique ? Voilà la question qui nous anime dans le contexte particulier qui est qu'Uzeste est devenu un territoire parmi tant d'autres de l'homo péri-urbanus, citoyen qui a évidemment d'autres chats à fouetter que d'aller spontanément participer à des manifestivités artistiques, surtout celles se proposant de réfléchir au fait que les têtes sont prises d'addictions salement salées que l'industrie du divertissement entretient savamment. Car l'art d'Uzeste, c'est du jamais-vu à la télé, du mal entendu à la radio, de la créativité écolo-artistique, sans OGM, conservateur ni colorant : du frais, du vrai, du singulier, de l'authentique de la liberté et de la responsabilité. Directement du producteur au spectateur sans intermédiaire! Sans rapport aucun avec les armes de distraction massive de la musique taylorienne. L'art que nous cherchons à créer « d'ici d'en bas » doit savoir avertir en divertissant, divertir en avertissant. Cet art d'avantgarde champêtre doit se nourrir de ce difficile équilibre instable, dialectique, entre ces deux nécessités humaines : s'avertir et se divertir, qui deviennent dans notre société marchandisante à outrance, antinomiques. Nous avons en effet essayé, et peut-être réussi, en certains espaces, d'instituer de cet art vital-vivant permettant que se créent des intervalles sociaux permettant de laisser respirer la pensée au-delà, en deçà des goûts et des couleuvres, des croyances, des appartenances et des suffisances. Nous avons participé à la naissance de nombreux festivals et cherché à essaimer par l'exemple. Ce que nous faisions à Uzeste était la preuve que l'on pouvait produire des choses en dehors des circuits marchands et depuis des lieux sur lesquels personne n'aurait misé. Par ailleurs, nous jouions souvent pour presque rien. La pratique poïélitique a toujours primé sur le cacheton et ça s'entendait. Nous jouions, « au noir ", un jazz imprégné du combat des musiciens blacks qui n'était pas notre combat à proprement parler, mais qui entretenait avec lui une évidente analogie. Le cri et la révolte qui portaient notre musique étaient semblables aux leurs, tout en étant différents car chargés de nos propres singularités, notamment territoriales. Nous avons joué sincèrement à nous jouer dans beaucoup d'endroits, en cherchant à faire la preuve de la possibilité d'une musique à vivre disant la nécessité d'inventer d'autres manières d'être ensemble au monde. Cette démarche nomadique d'autonomie et d'émancipation a nourri Uzeste qui a nourri cette démarche. Nous n'avons cessé de déterritorialiser- 
reterritorialiser nos pratiques en nous heurtant bien souvent à la trouille que produit la vision de la liberté, donc sans garantie de résultat.

9 F. G. - Tu parlais tout à l'heure d'intervalle. Uzeste pourrait sans doute être décrit comme un lieu excentrique à partir duquel s'élève une parole critique, où il est rendu possible de s'enrichir au partage et de se construire à l'échange en cultivant ce que François Jullien, dont la pensée est extrêmement proche de celle de Glissant sur ces questions, nomme des écarts. L'écart est ce qui permet de distinguer le commun de l'identique, ce qui permet de créer une distance et éventuellement de faire quelque chose de cette distance, d'initier l'exploration d'un possible. Le philosophe suggère, qu' a contrario de la différence, l'écart produit un dérangement, une prospection, un imprévisible. Le propre de l'écart est de maintenir en tension, à vif, à l'œuvre, de créer un entre-ouvert où "se déploie une intensité ». Il ajoute que " dans l'entre ouvert par écart, chacun, entrant en rapport avec l'autre, se dépossède de sa suffisance, déborde la clôture de son quant-à-soi " (2016: 39 et 74). J'imagine que ça résonne fortement avec la vision que tu as de vos activités « d'ici d'en bas »...

B. L. - Uzeste est effectivement un écart ou un intervalle ; un territoire intervallaire depuis lequel il est possible d'expérimenter des Relations qui te permettent d'inventer, qui t'invitent à prendre une certaine profondeur soumise à une problématique du "futur au présent" et qui ont justement vocation à refaire des histoires pour que l'histoire tire sur l'avenir. Je le déplore, mais aujourd'hui, à part nous, il n'y a pas trop d'histoires, ici. Nous essayons de faire des histoires, nous posons des actes qui UZinent des histoires, qui créent de la contradiction, qui créent de la pensée, du trouble, du tremblement, des écarts. Parmi les choses que l'on a peut-être réussie, il y a le fait que l'on ne passe pas pour des prétentieux, des donneurs de leçon, des " réussisseurs », des gagnants, des puissants. Dans la durée - quarante ans dans l'aidant - on a gagné notre position solidaire de rameurs qui ont plaisir/souffrir à se travailler et à produire de l'inconnu qui alerte sur notre connu, qui joue des préludes et des fugues de territoires que l'on a cernés et qui nous concernent. Dans l'ordre continental nous traçons des archipels qui mettent en lien des espaces de résistance : des territoires géographiques, politiques, philosophiques que l'on tente de faire dialoguer pour déconstruire les certitudes qui nous figent et nous abiment. Lors des Uzestivals ${ }^{1}$, par exemple, on essaie de donner une place importante au duel, au dual, aux intervalles où se risquent, l'art, la poésie, le politique, l'ici et l'ailleurs et qui nous rappellent à la nécessité du commun partagé et de son renouvellement permanent. Et durant les hestejadas, c'est identique. Une fois que c'est parti, c'est comme un concert improvisé : ça divague, ça se croise, ça s'emmêle, ça passe d'un territoire à un autre, ça transartistise.

11 F. G. - Dans une livraison des Annales de géographie dédiée au renouveau des fêtes et des festivals ( $\left.n^{\circ} 643,2005\right)$, Dominique Crozat et Sébastien Fournier notaient que nombre d'événements festifs s'inscrivaient, au début des années 2000, dans des territorialités puisant autant dans le local que dans le global. Il me semble que quinze années plus tard, leur constat reste d'actualité et que cette attirance pour un glocal souvent affadi s'est peut-être même renforcée. Les hestejadas de las arts pourraient sembler, à bien mal y voir, épouser cette logique postmoderne qui consacre in fine le hors-sol, la patrimonialisation déshistoricisée et le syncrétisme sauvage. Crozat et Fournier précisaient que ce local-global artificiel construit une hyper-réalité marketée qui n'est autre qu'un mélange de représentations et d'expériences sans liminalité et dont l'une des principales caractéristiques tient à sa conformité aux nécessités du 
marché mondialisé des loisirs et de la marchandise récréative standardisée. Ce qui se joue à Uzeste, où, comme tu aimes à le rappeler, la musique et l'art ne sont pas à vendre, mais à vivre, c'est évidemment tout autre chose. La mondialité plurielle qui traverse la localité uzestoise n'est pas la mondialisation marchande uniformisante ; elle se veut Tout-monde, elle est un concert baroque qui parie sur la mise en réseau des lieux, des espaces, des territoires intérieurs et extérieurs, tant symboliques que matériels.

B. L. - Comme je te l'ai dit, ce que l'on fait est venu se greffer sur un existant, mais sans lui être béatement fidèle. Les hestejadas sont, littéralement, des grandes fêtes des arts. Elles n'ont rien à voir avec les festivals tels qu'on les connaît aux alentours et ailleurs, qui diffusent de la musique en conserve pour consommateurs de boîtes. Il y a, par exemple, beaucoup de festivals qui font jouer du jazz d'il y a quarante ou cinquante ans, sans se préoccuper de ce qui arrive au jazz aujourd'hui, ni même de la manière dont cette transformation fait sens, parce que le jazz c'est un thermomètre. On trompe les gens en leur faisant écouter comment nous étions autrefois et en leur faisant croire combien c'était mieux. Pareil pour le rock'n'roll : une révolte de soixante-dix ans qui n'a pas bougé! C'est quand même vachement pratique pour la mise en conserve: "Achetez le disque, vous allez voir, c'est vraiment révolté, c'est super!». Où est l'homme révolté là-dedans ? Rejouer la tradition, c'est important, mais encore faut-il la réinventer, c'est-à-dire montrer des possibilités de jouer qui sont éminemment éduquantes, qui ont valeur de modèle en tant qu'elles ouvrent des possibles, non qu'elles reproduisent les pédagogies de la marche militaire. Il s'agit d'inventer, de mettre à l'œuvre des imaginaires, de désordonner les formes, mais évidemment ça n'est pas simple car il faut du désir, des compétences et se battre contre ceux qui ne veulent surtout pas te laisser faire, parce qu'ils voient très bien ce qu'ils ont à y perdre. Rejouer la tradition est donc bien un combat politique au fondement duquel on trouve la nécessité du décentrement du territoire, mais aussi de décentrement de soi, entre bocal, local, régional, mondial.

F. G. - Ce travail de décentrement ouvre à des formes de subjectivation que tu considères comme politiques et à des espaces publics de parole et d'action qui seraient des mondes communs, mais dont j'ai l'impression qu'ils se résument la plupart du temps à des espaces scéniques. Or des artistes en scène ne peuvent être, au mieux, que des communautés politiques singulièrement situées et éphémères. Si elles ouvrent, le temps d'un spectacle, à la possibilité pour chacun, ensemble, à des formes d'autogouvernement, celles-ci instituent un faire-commun qui n'a, a priori, aucune raison de déboucher sur un agir concerté donnant lieu à l'existence de mondes communs « obscène ». L'art ne saurait être qu'un ingrédient de ce que le philosophe Étienne Tassin nomme cosmopolitique et Glissant Tout-monde; il ne saurait en être ni la matière ni le lieu essentiels.

B. L. - Arriver dans des villages et jouer comme nous jouons, c'est comme si tu arrivais pour faire un meeting politique auquel personne ne s'attend. C'est comme tenir une réunion citoyenne du troisième type qui ne s'appuie pas sur des certitudes ou un programme, mais sur l'inconnu d'être ensemble, sur l'inexploré de la Relation, sur le risque et l'engagement à y aller sans être au point. Faire avec. Avec soi, avec les autres, ensemble. La musique à vivre est une autre manière de faire politique, via une représentation qui n'est pas délégataire, mais singulière. La représentation à l'œuvre c'est le spectacle des musiciens se jouant, s'instituant comme tu dis, dans le respect de 
l'autonomie de chacun. C'est un politique dont le territoire est la scène, fondé sur l'art de se faire des scènes de méninges.

F. G. - Tu dis souvent que l'art n'est plus dans notre culture. Aussi, il est un combat à mener pour faire prendre conscience de l'importance de l'art dans la société, de ces territoires esthétiques au principe du déploiement de l'imaginaire, c'est-à-dire de cette capacité d'inventer, de s'inventer, d'avoir conscience de ses limites pour pouvoir mieux les dépasser. Tu aimes beaucoup citer cet aphorisme de William Shakespeare : « Ils ont échoué parce qu'ils n'avaient pas commencé par le rêve ». En d'autres termes, l'art a partie liée à la poétique, c'est-à-dire une manière de se concevoir et de concevoir un rapport à soi, aux autres et au monde porté par des possibles: "L'imagination maintient en éveil le principe de plaisir en suggérant que l'accessibilité au bonheur n'est pas un rêve impossible. Face à la servitude imposée par le principe de réalité, la fonction critique de l'imagination consiste au moins à ce que les aspirations à la liberté et au bonheur ne soient pas oubliées » (Lachaud, 2015 : 130-131). Les "œuvriers» d'Uzeste sont des cultivateurs de culture et l'on pourrait peut-être même avancer qu'ils sont des cultivateurs d'imaginaires. Le devoir d'invention d'une ruralité critique que vous posez au principe de vos activités se traduit pour une large part, me semble-t-il, dans la tentative de construction d'imaginaires politiques territorialement ancrés, de ce que l'on pourrait désigner comme des imaginaires topolitiques.

B. L. - D'abord, ce que l'on essaie de nous faire croire, c'est que l'art ce n'est pas pour nous! C'est ce qu'on entend à peu près partout, et pas que dans les milieux populaires, relayé par les médias populo-commercialistes. Alors si ce n'est pas pour nous, c'est pour qui exactement ? Les nantis, les instruits, les privilégiés, les marginaux, les minorités dominantes, les pris de remords ? Bien sûr que l'art c'est nous, ça ne peut être que nous en tant qu'il est une représentation de toutes les activités sensibles, le rendu visible-audible-perceptible du pour qui-pourquoi-comment de notre condition humaine. L'histoire de l'art, de la culture, du politique, n'est pas le fait d'une entité tierce, mais bien le fait de tout un chacun, d'un imaginaire qui, comme le souligne André Breton, doit reprendre ses droits. Sauf qu'aujourd'hui, cet imaginaire, il est largement confisqué, cadré et déformé par les intérêts marchands et ceux qui cherchent à nous vendre des produits de substitution par lesquels on croit développer un style de vie, une singularité, alors qu'il s'agit évidemment d'un processus de conformation. L'art, c'est ce qui nous rend désirant, connaissant, pratiquant de nousmêmes, cultivateurs de nous-mêmes comme champs d'existence. Il s'agit d'inventer la vie, de s'inventer soi. Il faut s'imaginer, se convoquer au mensonge, à une autre forme de disponibilité, à une mémoire du futur et de l'imaginaire. Comme le dit Georges DidiHuberman, il y a dans notre façon d'imaginer, la condition de la manière dont nous pouvons faire de la politique. L'art permet de nous imaginer, d'inventer nos vies, nos avenirs, ce que nous voulons être et faire. Si nous ne prêtons pas assez d'attention à cette dialectique imaginaire/politique, c'est le passé mort qui figera la créativité du présent vivant.

17 F. G. - Votre démarche est, me semble-t-il, assez proche de ce qu'avec Cornélius Castoriadis on pourrait peut-être appeler un processus d'institution imaginaire des territoires - c'est-à-dire la faculté collective d'instituer des transformations sociales territorialisés -, processus essentiel à la création de territorialités nouvelles se jouant entre société instituée (histoire faite) et dynamiques instituantes (histoire se faisant). Ces imaginaires uzestiens dessinent en quelque sorte une carte mentale des terroirs et 
des territoires utopiques, des UZ-topies (Granjon, 2016), des mondes incréés comme dirait Glissant, c'est-à-dire des mondes à créer bien qu'ils soient déjà là, mais que l'on ne peut aborder qu'à l'aide des puissances de l'imaginaire et de l'intuition politique. D'ailleurs, la Compagnie Lubat n'est plus, aujourd'hui, "de Gascogne», mais "de Jazzcogne ». Glissant affirme que la mondialité est un sentiment imaginaire qui envisage la multiplication des diversités en les mettant en relation les unes avec les autres (2002: 77). La Jazzcogne ne serait-elle pas une forme de mondialité à la mode uzestoise ? Le terme ne dit-il pas, à sa manière, que le lieu particulier, aussi singulier et enraciné soitil ne peut plus, sauf à se racornir, se contenter d'être une unité fermée et close ? Glissant aimait rappeler que le lieu est incontournable dans sa localité (on ne peut en faire abstraction), mais il est également incontournable dans sa globalité, c'est-à-dire qu'on ne peut en faire le tour.

B. L. - La Compagnie Lubat est en effet jazz-concubine, c'est-à-dire licencieuse de l'imaginaire et licenciée de la doxa. La Jazzcogne est un territoire imaginaire, un territoire utopique, une UZ-topie comme tu dis. Elle est un territoire inventé, mais surtout à inventer et à réinventer, sans bords. Il n'existe pas matériellement, mais il a une influence sur tous les autres territoires, il fait des dégâts partout. Surtout, il nous donne une vision un peu trouble de tout le reste. C'est une loupe trouble, dé-polie, qui permet d'avoir à l'œil ce que nous ne pouvions/voulions entendre; de rendre visible, mais cette visibilité est brouillée, tremblée, incertaine. L'imaginaire de la Jazzcogne ce sont des points de vue qui troublent la vue et invitent à un déplacement du regard qui pose problème sans jamais apporter de solution. C'est une interpellation : des pouvoirs publics, des élus, des gens du village. C'est aussi ce qui permet de ne pas y croire, d'agir à penser, de penser à agir, sans croire qu'il y a des limites à l'exercice. À Uzeste, on joue à des je(ux) avec des individus qui sont à la fois territorialisés et déterritorialisés ; frontalement, sans répit et c'est l'imaginaire qui permet de rendre la confrontation supportable, qui rend possible cet art de la Relation qui n'est pas de tout repos. L'imaginaire UZ-topique, c'est aussi un territoire qu'il y a dans la tête des gens. Au bout de quarante ans, on a réussi ça tout de même: faire maintenant partie de l'histoire locale et des représentations qu'ont les gens du territoire, y compris chez ceux qui n'ont jamais foutu les pieds à L'Estaminet. C'est rentré dans la culture mais comme un brouillard. C'est diffus.

F. G. - Cet imaginaire diffus est un carrefour de territoires, de "topies" critiques visant une politique d'action à la base via des mobilisations croisées, à la fois esthétiques, sociales et théoriques dont le but est d'opérer ce que Bertolt Brecht présente comme la nécessité de rendre "étrange ce qui est familier» $(2000: 369)$ ou "de voir dans une chose ce qu'elle n'est pas, de la voir autre qu'elle n'est" (Castoriadis, 1999 : 177). C'est un espace de résistance qui se construit, volens nolens, sur des bases radicalement émancipatrices, par diverses formes d'expérimentation qui ont cependant du mal à cohabiter, car la recherche de nouvelles formes de vie suppose fatalement la mise en tension d'engagements, de sensibilités, de désirs qu'il s'agit d'organiser à une échelle locale dans des formes de coopération politique qui ne vont jamais franchement de soi. Notamment, sur le territoire, on a comme l'impression que se rejoue parfois comme une opposition entre nouveaux arrivants ou « néoruraux » qui poussent des revendications politiques depuis des types d'engagements variés, et Uzeste Musical qui défend pour l'essentiel une politique du sensible ouverte, mais qui 
tend tout de même à hiérarchiser les manière de s'investir pour faire de l'art la cause commune et première.

B. L. - Non, je ne crois pas. Il y a des écarts dans l'objet des mobilisations et donc forcément dans les moyens mis en œuvre, mais il n'y a pas d'opposition. C'est juste que les imaginaires que nous développons ont pour extravagance de passer par l'art. C'est par où ? C'est par l'art! À chacun sa "d'ici-dance» instituante. Et l'art est critique, aussi bien du nouvel arrivant que de l'ancien parvenu. L'écologiste, ici, il peut être très étonné, quand il est présenté aux chasseurs de palombes, de se retrouver face à des pratiques qu'il ne pensait pas telles qu'elles sont véritablement. Il va alors falloir qu'il pense à nouveaux frais. Et quand le chasseur lui expliquera très précisément sa culture de la chasse, il va falloir, de surcroît, qu'il s'interroge sur le fait que le savoir peut aussi venir d'une tradition, d'un terroir, d'une culture ancestrale: une connaissance pratique, pas seulement théorique. Aller à la rencontre d'Uzeste, c'est d'abord reconnaître cette richesse. C'est aussi prendre en considération le travail que nous y faisons depuis maintenant près de quarante ans et que nous avons réussi à faire reconnaître, y compris par ceux qui s'y opposent. Si tu veux prendre part et faire place pour des engagements, il n'y a, je crois, pas d'autre solution que de se greffer, au préalable, à l'existant par l'échange ; je ne vois pas d'autre possibilité. Il ne peut pas y avoir d'engagements conséquents à Uzeste sans la prise en compte de ce background, ainsi que la volonté parallèle de s'opposer au présentisme marchand qui organise cet oubli de l'histoire. Les prérequis sont donc nombreux car si le temps n'a pas d'âge, il laisse des traces, des images, des habitus que l'on ne peut pas ne pas prendre en compte. La transformation est difficile, on ne fait jamais table rase du passé. Pour que la foule esclave se mette debout, il faut qu'elle se relève en prenant appui sur l'histoire, sur certaines traditions, tout en en faisant la critique.

21 F. G. - L'art ne bouleverse pas le monde, mais encourage son éventuelle (r)évolution. Tu suggères que l'on pourrait se contenter des encouragements, l'important étant de se mettre en chemin, mais sans avoir à viser un point d'arrivée particulier et donc, par conséquent, sans avoir à organiser la stratégie pour atteindre cet objectif. Cette ouverture maximale à l'imprévu a toutefois pour faiblesse de ne pas prendre très au sérieux la dialectique de l'événement et de l'histoire, laquelle ne saurait être seulement rabattue sur un intérêt pour des formes de résistance de portée locale qu'il s'agirait d'articuler ex post afin de " changer le monde sans prendre le pouvoir » selon le slogantitre de l'ouvrage de John Holloway. N'est-ce pas se condamner à une impuissance fondamentale à faire converger les luttes, faute d'un intérêt minimal pour une forme d'universalisme stratégique? À trop insister sur la construction d'une sensibilité engagée - un imaginaire non résigné -, n'en vient-on pas à perdre de vue les aspects les plus collectifs de la lutte, en dehors même des tâches collectives assurant les conditions de possibilité de cet art critique? L'option de l'agrégation d'individus-sujets mis en relation à partir de subjectivités autonomes, d'identités sociales instables, négociables, traversées de signifiants flottants, ne me semble guère convaincant en termes d'organisation des luttes. Si l'on part du principe qu'il ne peut plus y avoir qu'« une multiplicité de conflits et d'actants juxtaposés, il n'y a [nécessairement] plus d'alternative d'ensemble à l'ordre social réellement existant » (Bensaïd, 2008 : 63). La résistance perd alors de sa charge agonistique et voit son caractère de classe, ou plus simplement collectif, être réduit à des réactions (inter)individuelles et instrumentées qui n'échappent pas toujours au narcissisme des petites et moyennes différences : le moléculaire, le détournement, les logiques affinitaires et la tactique personnelle, versus 
le molaire, l'organisation, la confrontation et la stratégie collective. La lutte contre le pouvoir qui assujettit devient, dès lors, l'affaire de singularités individuelles plus ou moins soustraites des rapports de force, de micro-résistances et de subjectivités alternatives, plutôt que de sujets socialement solidaires, politiquement mobilisés par un projet collectif de réponse à l'ordre social. Le risque est grand de voir émerger de nouvelles mythologies spontanéistes mettant en récit des résistances bricoleuses partant à l'assaut des remparts du capitalisme. Les trompettes de Jéricho, même revisitées par Miles Davis, Don Cherry ou Clifford Thornton n'y suffiraient pas. Si, comme le suggère LeRoi Jones, le blues primitif est l'expression de l'individualité du Noir au sein de la communauté américaine, tu conviendras que la libération de l'esclavage et de la ségrégation raciale ne s'est pas réalisée seulement en chantant des Work songs!

B. L. - Mais j'en conviens. L'art est une forme de résistance populaire minoritaire. D'ailleurs, les gens ne savent pas toujours à quoi nous résistons, ici. Certains sentent que nous résistons à quelque chose, une minorité se doute que nous résistons sans trop savoir à quoi exactement. Il y a une sorte d'acceptation de cette indétermination. Nous sommes acceptés en tant que mythomanes, ceux qui racontent des histoires, qui font de la musique qui gueule, qui dérange. Parce que nous leur avons fait croire, ici comme ailleurs, que la musique c'est forcément de la chanson, de la musique radioactive vingtquatre heures sur vingt-quatre. Et puis quand ce n'est pas de la chanson, c'est de la musique d'ambiance pas très forte, même si c'est Charlie Parker dans la salle d'attente du dentiste ou Robert Schumann dans le parking. C'est une façon de remettre la musique à sa place. On exploite la musique, on la chosifie, réifie, elle devient le fétiche des oreilles, un son industriel mis en boîte - à rythme - que l'on essaie de nous vendre et de naturaliser comme étant de la bonne musique. On organise le mal-ouïr comme la malbouffe, par la profusion : la musique, il n'y en a plus parce qu'elle est partout et c'est partout la même. L'art musical advient quand c'est louche à l'oreille, quand ce n'est pas clair et que l'on se demande : «Qui suis-je, moi, là-dedans? Où sont mes goûts et mes couleurs? Qu'est-ce que j'en pense ? Qu'est-ce que c'est que ce truc-là ? ». C'est à partir du moment où l'on se pose ces questions que l'on se soigne de la malouïr, que l'on peut imaginer le futur de la musique, car il y en a autant à faire que ce qui a déjà été fait. C'est ce qui m'intéresse dans cette audition du futur : l'inconnu, ce que l'on ne sait pas de soi. À Uzeste, nous déplaçons la musique, nous faisons de la musique déplacée, inattendue, en mouvement, au fur et à démesure, qui permet peut-être de se libérer des effets nocifs du parachutage des goûts et des couleuvres radioactifs. Les gens sont atteints d'une addiction terrible : il y a ceux qui s'en rendent compte, ceux qui ne s'en rendent pas compte et pour moi, être politique c'est d'abord se battre contre ça avec les armes de l'art, c'est-à-dire un gros point d'interrogation offensif, actif : faire en sorte que les gens soient devant cet art et se disent : « Mais qu'est-ce que j'en pense de ça ?». Se battre pour que l'on pense.

F. G. - Il ne fait donc aucun doute que, pour vous, l'art est l'outil privilégié d'arpentage des territoires de l'autotransformation et qu'il invite à interroger, d'une part, au niveau individuel, les territoires du transcendantal (l'imagination radicale du sujet), et, d'autre part, à un niveau collectif (l'imaginaire social instituant), les dynamiques territorialisées de conduite $d u$ changement social. L'art serait donc un ingrédient nécessaire à l'émergence d'espaces d'expérience, de réflexivité et d'expérimentation, 
autrement dit d'espaces politiques testant d'autres formes de démocratie "par en bas".

B. L. - Aux hauteurs, je préfère les profondeurs. S'approfondir plutôt que s'élever. Et pour forer convenablement, il faut sans arrêt se mettre des bâtons dans les Nous. Il faut travailler à son exception, à sa singularité; travailler à se donner l'autorisation de se jouer, de se risquer à soi, de se confronter à soi-même. Et l'instrument privilégié pour arpenter les terres inconnues de soi et de la Relation, c'est l'improvisation à égalité. L'improvisation est une démocratie qui permet de s'appliquer à jouer comme personne. On me demande parfois pourquoi je ne joue pas comme les autres. Parce que les autres s'en occupent!

F. G. - Dans Rues de Berlin et d'ailleurs, Siegfried Kracauer affirme que « La valeur des villes se mesure au nombre des lieux qu'elle réserve à l'improvisation » (2013:89). Si l'on prend au sérieux cet aphorisme, Uzeste n'a certainement pas de prix, mais assurément de la valeur dans la mesure où il y est des lieux, à commencer par L'Estaminet, où ça ne cesse d'improviser pour sortir des « cadences » de la musique et de la vie, pour faire en sorte que la vie administrée puisse se fissurer et ouvrir les voies à des imaginaires et des actions alternatifs. Tu as parfois qualifié l'improvisation d' esthétique des conjugaisons, non en ce qu'elle serait le moyen d'une esthétisation stylistique de l'existence, mais dans le sens où elle serait une forme d'agir permettant d'envisager le soi comme une structure éthique de personnalité à l'œuvre (Granjon, 2018). Non pas une subjectivité préalablement définie et vers laquelle il faudrait tendre ou qu'il faudrait retrouvée - fut-elle critique -, mais plutôt un processus de subjectivation à conquérir en permanence sur le prescrit, l'attendu, le tolérable et leurs contradictions. L'improvisation telle que tu la pratiques apparaît donc comme un cadre pour se devenir et s'apprendre. C'est une éducation à l'individuation intranquille, celle d'avoir à produire ses propres (impro)visions ; l'intranquillité d'avoir à détruire en soi ce qui nous rappelle à l'ordre, d'avoir à s'exiger un impossible présumé que l'on croit hors de portée et donc à s'engager.

B. L. - Uzeste est un laboratoire pour un art impopulaire, d'avant garde, expérimental, biodégradable. Nous sommes des joueurs, on joue à jouer, à se jouer. Nous sommes des renégats car nous ne voulons pas nous laisser simplifier. Nous nous improvisons nousmêmes tel que nous l'entendons, si nous nous entendons. L'improvisation, encore une fois, c'est la relation au sens de Glissant, la rencontre avec des individus qui sont eux aussi des territoires et sont dans des territoires qui leur font jouer des rôles. Untel est dans son territoire d'élu quand il nous invite à son évènement départemental, mais je le connais aussi dans son personnage du marché de Bazas, un autre territoire. L'improvisation c'est un moyen de lever les frontières des territoires de chacun. Avec Serge Pey que je connais, que j'ai lu, j'ai passé la semaine précédant notre rencontre ${ }^{2}$ à me demander comment j'allais pouvoir jouer avec ce qu'il est, avec ce que j'imaginais être sa réaction. Avec Richard Bohringer, même chose ${ }^{3}$ ! J'ai pensé que les archétypes du blues nous permettraient de nous rencontrer plus facilement, puis en jouant, je me suis rendu compte qu'il allait falloir négocier tout autrement. L'improvisation est l'agir au travers duquel le passage se propose. C'est ce que l'on fait qui nous mène. Je ne joue pas, je suis joué par la relation à l'autre. C'est un tremblement où s'invite de l'invention et la liberté de passer d'un territoire à un autre, avec des droits de douane, des douanes de gauche. Si l'on s'en tient à la musique, comme je suis un raté de la classe de musique classique, un défroqué de la variété et un sous-pape du jazz, ce qui me sauve c'est de 
pouvoir improviser avec tout ce merdier : passer d'une île à une autre, tracer des pistes dans l'archipel de mes connaissances-ignorances.

F. G. - On pourrait effectivement penser que ce que vous construisez, depuis plus de quarante ans maintenant, relève de phénomènes d'archipélisation. Chez Glissant, l' archipel est une figure du décentrement et il me semble que votre rapport aux territoires tant géographiques qu'intérieurs est précisément un rapport d'excentration : partir certes d'un point, mais pour tracer de multiples lignes de fuite. Dans l'ouvrage intitulé L'île déserte, Gilles Deleuze écrit : «Rêver des îles, avec angoisse ou joie peu importe, c'est rêver qu'on se sépare, qu'on est déjà séparé, loin des continents, qu'on est seul et perdu - ou bien c'est rêver qu'on repart à zéro, qu'on recrée, qu'on recommence ». Et il ajoute : «Séparation et recréation ne s'excluent pas sans doute, il faut bien s'occuper quand on est séparé, il vaut mieux se séparer quand on veut recréer, reste qu'une des deux tendances domine toujours" (2002: 11-12). N'est-ce pas là un moyen d'incuber des liens susceptibles de refonder, au moins partiellement, l'intersection entre l'individuel et le collectif, le privé et l'étatique, le personnel et le/l' (inter)communal, le social et le politique, et de dessiner ce que Christos Giovanopoulos désigne comme "une nouvelle topographie des relations sociales et de la subjectivation politique » $(2018: 102)$ ?

B. L. - Uzeste, c'est lîle des " comment-se-ment » où l'on échoue quand on a réussi à larguer les amarres de la cadence et de la déconfiance en soi ; une île depuis laquelle on peut travailler à avoir de nouveau « conscience en soi » et à ne plus avoir la trouille d'être libre. La pensée et l'agir-archipel permettent de passer d'une île à une autre, de la séparation à la recréation.

F. G. - Une dernière chose : Agnès Varda, récemment décédée, éminente documenteuse qui est notamment venue en 2000 à Uzeste, en soutien à votre grève, projeter Les Glaneurs et la Glaneuse; réalisatrice dont le documentaire qu'elle a tourné avec le plasticien JR s'intitule Visages Villages (2017), rappelant ainsi le nom même de votre association "Uzeste Musical, visage village des arts à l'œuvre "; Agnès Varda dont le générique du superbe film Les Plages d'Agnès (2008) n'est autre que Loulou Zoulou tiré de ton album Chansons enjazzées (2008); Agnès Varda donc... uzestienne en diable et en bien des aspects de sa démarche et de sa personne, disait : "Si on ouvrait des gens, on trouverait des paysages. Si on m'ouvrait moi, on trouverait des plages ». Et toi, si on te décachetait, on trouverait quel territoire?

B. L. - Pas une plage, c'est sûr... On trouverait certainement une forêt sauvage avec ses êtres. Le territoire feuillu d'une polyrythmie lente, à l'opposé du temps hystérisé des Macdominants. La forêt est un intervalle complexe qui incite à être à l'affût de soi, à être attentif à la nécessité de soi-même. C'est l'espace des profondeurs et des complexités, à la fois apaisant et intranquillisant. On ne sait jamais ce qui va sortir du bois... comme de soi. 


\section{BIBLIOGRAPHIE}

Bensaïd (Daniel), Penser agir, Paris, Lignes, 2008.

Brecht (Bertolt), Écrits sur le théâtre, Paris, Gallimard, 2000.

Castoriadis (Cornélius), L'institution imaginaire de la société, Paris, Seuil, 1999.

Crozat (Dominique), Fournier (Sébastien), « De la fête aux loisirs : évènement, marchandisation et invention des lieux », Annales de géographie, n 643, 2005, pp. 307-328.

Deleuze (Gilles), L'île déserte. Textes et entretiens 1953-1974, Paris, Éditions de Minuit, 2002.

Giovanopoulos (Christos), « La Grèce après l'espoir : en attendant le possible. Réflexions sur le mouvement des solidarités locales », Vacarme, nº 83, 2018, pp. 99-108.

Glissant (Édouard), «La relation, imprédictible et sans morale - Entretien avec François Noudelmann », Rue Descartes, n 37, 2002, pp. 76-95.

Granjon (Fabien), «Improvisation et Parrêsia. Des pratiques politiques de soi », in Denouël (Julie), Granjon (Fabien) dir., Politiques d'UZ : vivacités critiques du réel, Rennes, Éditions du commun, 2018, pp. 145-188.

Granjon (Fabien), Les UZ-topies de Bernard Lubat (Dialogiques), Paris, Outre Mesure, 2016.

Holloway (John), Changer le monde sans prendre le pouvoir. Le sens de la révolution aujourd'hui, Paris/ Montréal, Syllepse/LUX, 2008.

Jullien (François), Il n'y a pas d'identité culturelle, Paris, L'Herne, 2016.

Kracauer (Siegfried), Rues de Berlin et d'ailleurs, Paris, Les Belles Lettres, 2013.

Lachaud (Jean-Marc), Ce que peut (malgré tout) l'art, Paris, L'Harmattan, 2015.

Laval (Christian) et al., dir., L'alternative du commun, Paris, Hermann, 2019.

Nicolas-Le Strat (Pascal), Le travail du commun, Rennes, Éditions du commun, 2016.

\section{NOTES}

1. Les Uzestivals sont des festivals saisonniers. http://www.uzeste.org/manifestivites/uzestivalde-printemps-2019/.

2. https://fabiengranjon.eu/recital-bernard-lubat-serge-pey-regarder-passer-les-trous-desoiseaux-uzeste-5-janvier-2019/.

3. https://fabiengranjon.eu/rencontre-blues-de-la-richard-bohringer-bernard-lubat/. 